\title{
The Effect of Maternal Caffeine Ingestion on Pancreatic Function in the Neonatal Rat
}

\author{
M. Dunlop ${ }^{1}$, R. G. Larkins ${ }^{2}$ and J. M. Court ${ }^{1}$ \\ ${ }^{1}$ Department of Developmental Paediatrics, Royal Children's Hospital, Parkville and \\ ${ }^{2}$ Department of Medicine, University of Melbourne, Repatriation General Hospital, Heidelberg, Victoria, Australia
}

\begin{abstract}
Summary. Pancreatic function was investigated in neonatal suckling offspring of caffeine-ingesting dams, with or without maternal sucrose supplementation, throughout pregnancy and lactation. In offspring of rats ingesting caffeine without sucrose supplementation, there was initial hyperinsulinaemia, followed by a progressive fall of plasma insulin to subnormal levels. This fall in plasma insulin coincided with depletion of pancreatic insulin stores. Both the fall in plasma insulin and depletion of pancreatic insulin stores were prevented by sucrose supplementation of caffeine-ingesting dams. Offspring of dams fed sucrose alone and control offspring also maintained pancreatic insulin stores and circulating insulin levels over the first 14 days of postnatal life. Pancreases from offspring of caffeine-exposed animals tested in vitro showed enhanced sensitivity of the insulin release process to glucose. This was reflected in the glucose concentration required to elicit half-maximal insulin release ( $2.4 \pm 0.2 \mathrm{mmol} / \mathrm{l}$ for caffeine offspring, $2.3 \pm$
\end{abstract}

$0.2 \mathrm{mmol} / \mathrm{l}$ for caffeine with sucrose, $3.8 \pm 0.3 \mathrm{mmol} / \mathrm{l}$ for sucrose and $4.1 \pm 0.3 \mathrm{mmol} / \mathrm{l}$ for control offspring, mean \pm SEM). In contrast, offspring of sucrose-supplemented (with or without caffeine) dams showed increased sensitivity of the proinsulin biosynthetic process to glucose, whereas offspring of dams ingesting caffeine alone showed no significant enhancement of the biosynthetic process compared with control offspring. Thus enhanced sensitivity of the insulin secretory process to glucose without a change in the sensitivity of the biosynthetic process in the offspring of the caffeine ingesting (nonsucrose supplemented) dams could explain the progressive depletion of pancreatic insulin stores and eventual hypoinsulinaemia seen in this group.

Key words: Caffeine, neonatal rat, sucrose supplementation, pancreatic insulin, insulin release, proinsulin biosynthesis.

the offspring weight reduction attributed to caffeine exposure was not found after introduction of supplementary carbohydrate, as sucrose, to the maternal diet. This dietary supplementation resulted in increased offspring birth weight and growth [14]. These findings are of particular interest as human consumption of caffeine is often accompanied by sucrose ingestion.

The aim of the present investigation was to determine whether habitual low dose caffeine consumption by the mother affected the processes of insulin biosynthesis and release in neonatal rats and to examine the extent to which this was modified by concurrent maternal sucrose supplementation.

\section{Materials and Methods}

Caffeine was obtained from Sigma Chemicals, Clayton, Victoria, Australia. Mecon Nafag formulation rat and mouse diet is a pelleted food supplying $3040 \mathrm{Cal} / \mathrm{kg}$ dry weight with vitamin and trace element supplementation, obtained from Bunge, Melbourne, Australia. Rat insulin standard (Lot R177 $20 \mathrm{IU} / \mathrm{mg}$ ) was purchased from Novo Industri, Denmark; Eagle's minimum essential medium, amino acids $100 \times$ concentrate and McCoy $5 \mathrm{~A}$ medium (glucose free) prepared as a special product were purchased from the Commonwealth Serum Labora- 
tories, Melbourne, Australia; Instagel multicomponent scintillator was from Packard Instrument Company, Illinois USA; L-[4-5- $\left.{ }^{3} \mathrm{H}\right]$-leucine, $3.7 \times 10^{10} \mathrm{~Bq} / \mathrm{mmol}$ was from the Radiochemical Centre, Amersham, UK; guinea pig anti-insulin serum was from Wellcome Laboratories, Beckenham, UK. All other chemicals were of reagent grade.

Female rats of Wistar strain (180-200 g) were fed ad libitum with a commercial diet (Mecon Nafag, Bunge Mulgrave, Victoria, Australia). After mating with Wistar strain male breeding pairs, they were assigned to one of four dietary groups. In group 1 (caffeine), females consumed caffeine in amounts of $10 \mathrm{mg} \cdot \mathrm{kg}^{-1} \cdot$ day $^{-1}$ supplied in the drinking water. In group 2 (caffeine/sucrose), this caffeine solution was supplemented with sucrose so that $7 \mathrm{~g} \cdot \mathrm{kg}^{-1} \cdot$ day $^{-1}$ sucrose was consumed together with the caffeine. Group 3 (sucrose) received sucrose alone $\left(7 \mathrm{~g} \cdot \mathrm{kg}^{-1} \cdot\right.$ day $\left.^{-1}\right)$ in drinking water. Group 4 (control) received pelleted diet and water ad libitum. Animals obtained pelleted food through stainless steel troughs which prevented food access to preweaned offspring. Food consumption was determined daily. It has been shown previously that there was no significant difference in the pelleted food intake of groups maintained in this manner. The daily food consumption of $9.4 \pm 2.8 \mathrm{~g} / 100 \mathrm{~g}$ body weight (mean \pm SD) indicated adequate nutritional intake for all groups. Animals were maintained for sequential pregnancies, each terminating with spontaneous delivery on day 22 after mating, according to a protocol described previously [13]. Animals were weighed at the beginning of the study (nongravid) and immediately following parturition of each litter.

\section{Maternal Studies}

Two days before expected parturition dams were bled from a tail vein. Animals were not fasted and sampling in all cases was at $1200 \mathrm{~h}$. Plasma glucose was estimated using an hydrogen peroxide specific electrode employing glucose oxidase (Yellow Springs Instruments glucose analyser) and plasma insulin determined by radioimmunoassay with rat insulin standards [15]. The litters chosen for study were from the third pregnancy of caffeine-ingesting dams. In these litters neonatal offspring growth changes were first apparent [14]. Pups to be investigated were removed without disturbance to lactation or nurturing behaviour of the mother. Litter size was adjusted to $8-10$ pups after birth.

\section{Offspring Studies}

Male neonatal animals were killed by decapitation on each of the first 6 post-natal days and on day 14 after birth. Blood was collected from the cephalic return flow into heparinised tubes for the estimation of plasma glucose and insulin. Pancreases were dissected as completely as possible from the day of birth (designated postnatal day 0 ) for the first 6 days and on day 14 for estimation of weight and insulin content. In addition, on day 3 the splenic lobe of the pancreas was removed, transferred into a Petri dish containing ice-cold McCoy $5 \mathrm{~A}$ medium with glucose $(1.67 \mathrm{mmol} / \mathrm{l})$ and essential amino acids $(4.7 \mathrm{mmol} / 1)$ and was sectioned into $200 \mu \mathrm{m}$ slices for experiments in vitro. Islets were microdissected from separate splenic lobes and stored at $-20{ }^{\circ} \mathrm{C}$ for insulin determination.

\section{Immunoreactive Insulin Released in Response to Glucose}

Sections of the splenic lobe of the pancreas were incubated at $37^{\circ} \mathrm{C}$ under $95 \% \mathrm{O}_{2}, 5 \% \mathrm{CO}_{2}$ in medium containing 21 amino acids as described by McCoy et al. [16]. The amino acid concentration was further supplemented to $10 \mathrm{mmol} / \mathrm{l}$ with the essential and non-essential amino acids found in the near-term fetal rat aminogram [17]. The concentration of glucose was adjusted between 1.6 and $16.7 \mathrm{mmol} / 1$ by the addition of $\mathrm{D}$-glucose in a final incubation volume of $1.0 \mathrm{ml}$. After incubation for $60 \mathrm{~min}$ at $37^{\circ} \mathrm{C}$ aliquots of medium were removed for insulin determination. Results for insulin release were expressed as $\mu \mathrm{U}$ released per five islets.

\section{${ }^{3}$ H-Leucine Incorporation into Immunoprecipitated Proinsulin}

Separate pancreatic slices were incubated in the same medium at glucose concentrations of 1.6 to $16.7 \mathrm{mmol} / 1$. Tritiated leucine, $(15 \mu \mathrm{Ci}$ $\mathrm{L}-4,5-{ }^{3} \mathrm{H}$-leucine) was added and the incubation continued for $30 \mathrm{~min}$ in a final volume of $750 \mu \mathrm{l}$. After this time explants were washed with cold medium ( $1.6 \mathrm{mmol} / \mathrm{lglucose}$ and $4.7 \mathrm{mmol} / 1 \mathrm{amino}$ acids $)$ and incubated in glucose-free medium with leucine in excess for $15 \mathrm{~min}$ [18]. Pancreatic slices were removed, blotted on filter paper and sonicated individually in $0.1 \mathrm{~mol} / 1$ borate buffer ( $\mathrm{pH} 8.0$ ) containing $5 \%$ bovine serum albumin and $0.5 \mathrm{~mol} / 1 \mathrm{NaCl}$. Aliquots of this preparation were immuno-precipitated with an excess of guinea pig anti-insulin gamma globulin. After $12 \mathrm{~h}$ at $4{ }^{\circ} \mathrm{C}$, the antibody complex was precipitated by cold polyethylene glycol (molecular weight $3000-4000$ daltons, $12.5 \mathrm{~g}$ / $100 \mathrm{ml}$ final concentration). Following centrifugation the precipitate was sonicated with a minimum volume of ethanol: water $(7: 3, v / v)$ before addition to scintillant (Instagel). Radioactivity was measured with a counting efficiency of $32 \%$ for tritium. Results for tritium incorporation were expressed as $\mathrm{fmol}^{3} \mathrm{H}$-leucine incorporated per five islets.

\section{Pancreatic and Medium Insulin Content}

Freshly dissected pancreatic tissue micro-dissected islets and pancreatic slices with remaining medium from individual release experiments were homogenized at $4^{\circ} \mathrm{C}$ with the addition of sucrose containing medium to a final sucrose concentration of $0.15 \mathrm{~mol} / \mathrm{l}$. These homogenates and those obtained after homogenization of ${ }^{3} \mathrm{H}$-leucine containing pancreatic slices were diluted $1: 1000$ for estimation of insulin by radioimmunoassay [15]. The sensitivity of the assay allowed the detection of $3.7 \mathrm{mU} / \mathrm{l}$ in samples of $25 \mu \mathrm{l}$ volume. At 15 and $120 \mathrm{mU} / \mathrm{l}$ the intra-assay coefficients of variation were $4.7 \%$ and $2.7 \%$, and the interassay coefficients of variation were $6.4 \%$ and $5.4 \%$, respectively. The islet content of the pancreatic slices used for the secretion and biosynthesis studies was calculated from the value of insulin content of the slice compared to the average islet insulin content of the corresponding group of offspring. Results are expressed as mean \pm SEM for the indicated number of determinations. Student's t-test was used for statistical analysis of significance.

\section{Results}

The daily pelleted food intake of the pregnant dams was not significantly different: mean ( \pm SEM) daily food consumption over the entire pregnancy of $23.2 \pm 0.6$, $22.4 \pm 0.6,22.2 \pm 0.6$ and $22.0 \pm 0.5 \mathrm{~g}(n=126-168$ daily observations in each group) for caffeine, control, caffeine/sucrose and sucrose dams respectively. The sucrose intake could therefore be considered a hypercaloric supplementation. These diets were associated with weight gains, pre-pregnant to parturition of the study litter of $50 \pm 12,67 \pm 10,95 \pm 14$ and $104 \pm 14 \mathrm{~g}$ for caffeine, control, caffeine/sucrose and sucrose animals respectively: caffeine/sucrose and sucrose dams were significantly different compared with caffeine and control $(p<0.01)$. Pre-parturition plasma glucose and insulin values are shown in Table 1 . Circulating glucose was increased in animals receiving sucrose supplementation and plasma insulin was significantly increased in both groups of animals receiving caffeine.

Figure 1 indicates that over the first 6 post-natal days the expected temporal increase in pancreatic insulin content was seen in the control offspring. This was seen also in the pancreases from the caffeine/sucrose and 
Table 1. Maternal post-prandial plasma insulin and glucose levels measured 2 days before parturition of litter 3

\begin{tabular}{cccccc}
\hline \multicolumn{5}{c}{ Maternal diet } \\
\cline { 2 - 6 } & $\begin{array}{l}\text { Caffeine }+ \text { Sucrose } \\
\text { sucrose }\end{array}$ & Caffeine & Control \\
\hline $\begin{array}{c}\text { Plasma insulin } \\
(\mathrm{mU} / \mathrm{l})\end{array}$ & $94 \pm 4^{\mathrm{b}}$ & 80 & \pm 12 & $98 \pm 12^{\mathrm{a}}$ & $55 \pm 10$ \\
$\begin{array}{c}\text { Plasma glucose } \\
(\mathrm{mm})\end{array}$ & $7.4 \pm 0.7^{\mathrm{b}}$ & $\left.7.1 \pm 0.8^{\mathrm{b}}\right)$ & $5.4 \pm 0.6$ & $5.8 \pm 0.7$. \\
\hline
\end{tabular}

Values shown are mean \pm SEM with the number of mothers in parentheses.

${ }^{\mathrm{a}} p<0.05$ and ${ }^{\mathrm{b}} p<0.01$ indicate significant differences from control group

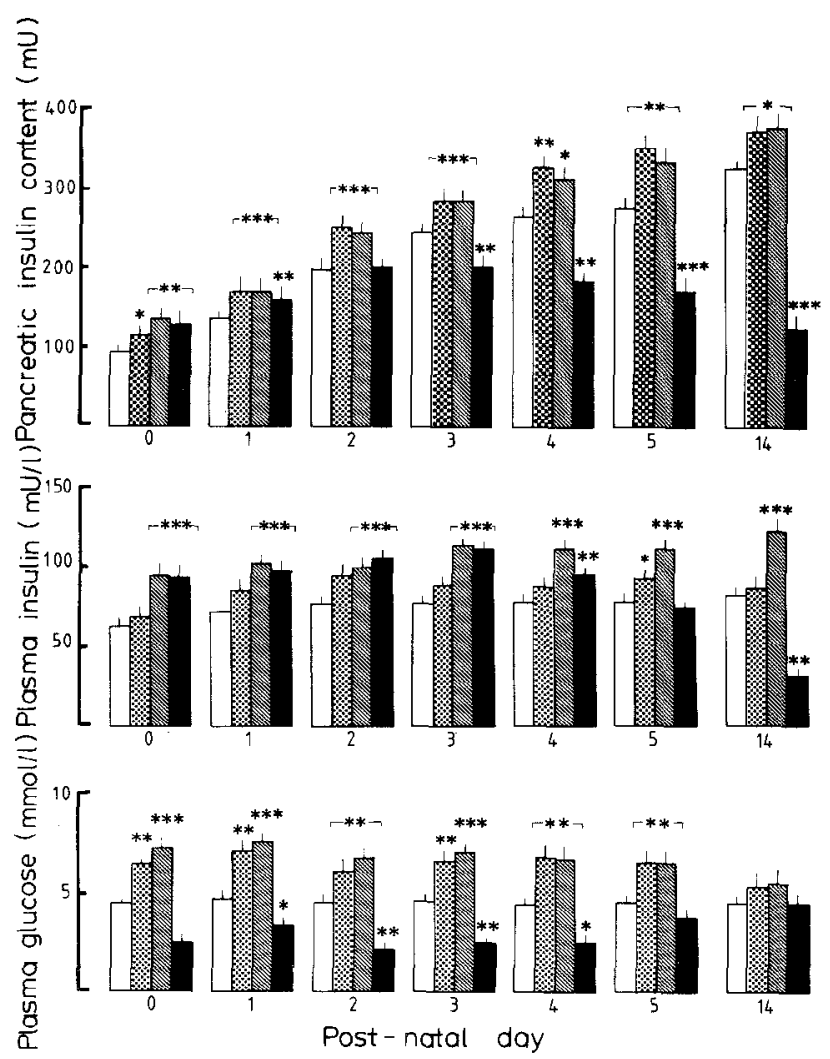

Fig. 1. Total pancreatic insulin, plasma insulin and plasma glucose in neonatal rats. Values shown are mean \pm SEM $\square$ control (six to eight offspring for each determination); 图 sucrose (eight offspring for each determination); caffeine/sucrose (eight offspring for each determination) and $\square$ caffeine (eight offspring for each determination). $* p<$ $0.05 ; * * p<0.01$ and $* * * p<0.005$ indicate significant differences from control group

sucrose offspring with significant increases in the insulin content at each age studied. In the caffeine group, an increased insulin content was demonstrated for the first 2 post-natal days, but insulin content then decreased and by the sixth post-natal day was significantly less than the control group. A further decrease was seen on the fourteenth post-natal day. The circulating insulin levels found in suckling animals are also shown in Figure 1. Both caffeine groups had significantly elevated plasma insulin levels for the first 4 days after birth. Plasma insulin levels of sucrose offspring were not significantly dif-

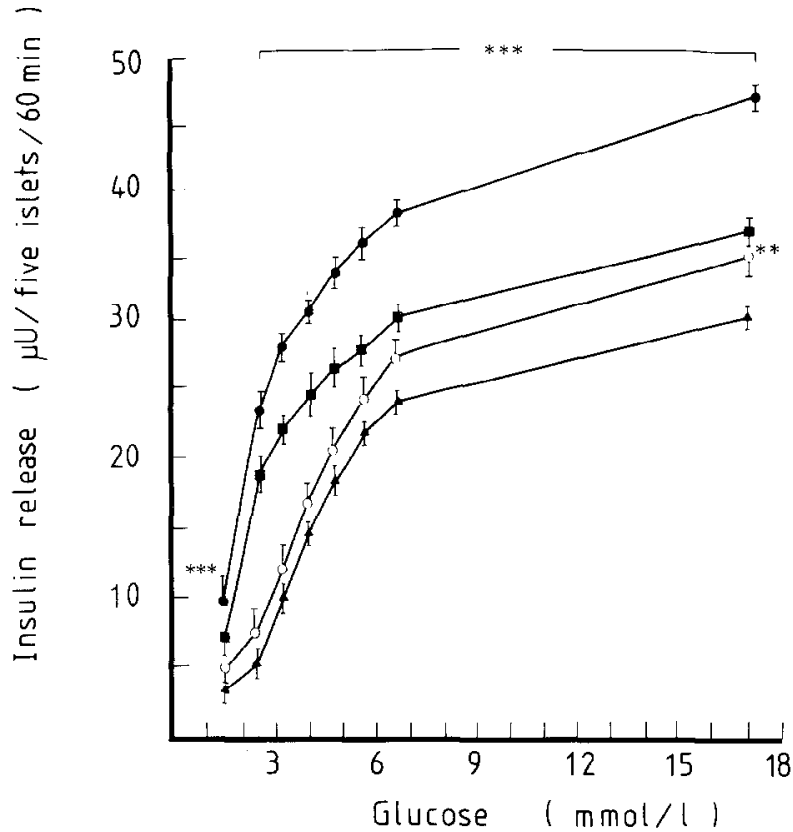

Fig. 2. Immunoreactive insulin released in response to increasing glucose concentrations in vitro in pancreas of neonatal rats on post-natal day 3. Values shown are mean \pm SEM for offspring of maternal diets; - caffeine/sucrose (eight determinations); caffeine (eight determinations); $\circ$ sucrose (eight determinations) and $\boldsymbol{\Delta}$ control (six to eight determinations). ${ }^{* *} p<0.001$ and ${ }^{* * *} p<0.005$ : the statistical significance of difference between observations and control. The bar indicates the significance of caffeine/sucrose and caffeine observations compared with control. Statistically significant differences between caffeine/sucrose and caffeine determinations are seen at 2.4, 3.2, 4.0, $4.8,5.6,6.4$ and $16.7 \mathrm{mmol} / 1$ ( $p<0.005$ in all cases) and between caffeine/sucrose and sucrose at all glucose concentrations $(p<0.005)$

ferent from control at this time. Caffeine/sucrose, sucrose and control offspring maintained circulating insulin levels whilst offspring of dams ingesting caffeine alone had progressively falling insulin levels so that by day 14 they were less than half control levels. The corresponding plasma glucose levels show that over the first 6 post-natal days caffeine/sucrose and sucrose offspring had significantly increased plasma glucose levels but the values of caffeine offspring were significantly reduced when compared with both control and sucrose supplemented offspring. Weight gained from birth to post-natal day 14 was $9.3 \pm 0.9,17.6 \pm 1.3,21.5 \pm 1.4$ and $22.7 \pm$ $1.9 \mathrm{~g}$ for caffeine, control, caffeine/sucrose and sucrose offspring, respectively.

Dose response curves for glucose stimulated insulin release are shown in Figure 2. Pancreatic tissue from both caffeine groups exhibited markedly enhanced insulin release in response to glucose with a further significant difference between the stimulated response of caffeine/sucrose and caffeine offspring. At the lowest glucose concentration used, there was a doubling of insulin release from pancreatic slices exposed to caffeine in vivo. At a glucose concentration of $16.7 \mathrm{mmol} / 1$, there was a significant increase in the insulin released from the pancreas of both caffeine groups. Glucose sensitivity was demonstrated by a threshold for release between 1.6 and $2.4 \mathrm{mmol} / 1$ glucose for control and sucrose pan- 


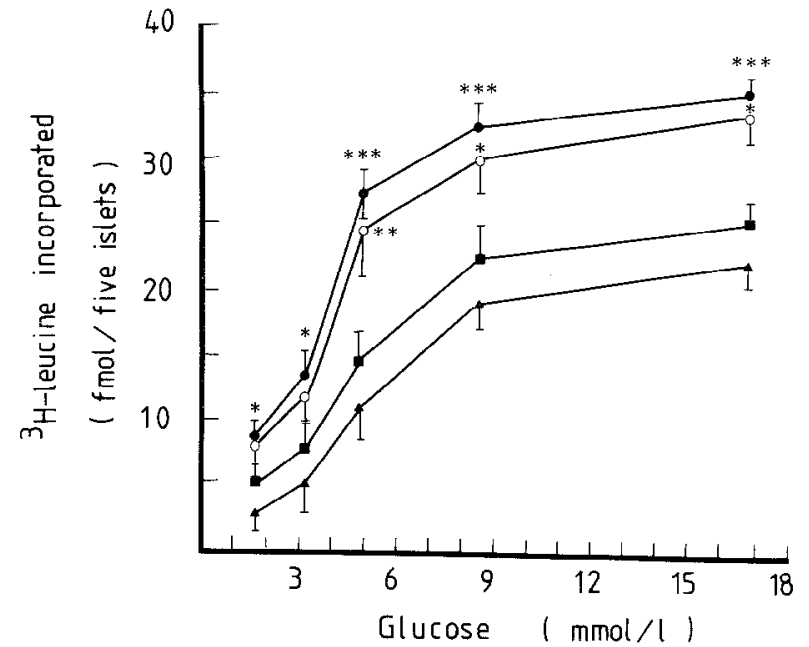

Fig. 3. Incorporation of ${ }^{3} \mathrm{H}$-leucine into proinsulin in the pancreas of neonatal rats on post-natal day 3 . Results are expressed as a function of increasing glucose concentration in vitro. Study groups and observation number are identical to those of Figure $2 . * p<0.05 ; * * p<0.01$ and ${ }^{* * *} p<0.005$ indicate statistical significance of difference between observation and control group. Statistically significant differences between caffeine/sucrose and caffeine determinations are seen at glucose concentrations $3.2 \mathrm{mmol} / 1(p<0.05), 4.8 \mathrm{mmol} / 1(p<0.01)$, $8.4 \mathrm{mmol} / \mathrm{l}(p<0.05)$ and $16.7 \mathrm{mmol} / 1(p<0.005)$

creases. Control and sucrose pancreases showed similar glucose responsiveness at physiological glucose concentrations. A significant difference was seen in the insulin released by the sucrose pancreases only at elevated glucose concentrations. The calculated value for $50 \%$ of maximal response for caffeine and caffeine/sucrose pancreases was found to be within this range of glucose concentrations $(2.3 \pm 0.2$ and $2.4 \pm 0.2 \mathrm{mmol} / 1$ glucose for caffeine and caffeine/sucrose, respectively). These values represent a significant shift in the sensitivity of the release process when compared with the calculated values for $50 \%$ of maximal response in sucrose and control pancreases which were $3.8 \pm 0.3$ and $4.1 \pm 0.3 \mathrm{mmol} / 1$ glucose respectively $(p<0.001)$.

The incorporation of ${ }^{3} \mathrm{H}$-leucine into proinsulin is shown in Figure 3. At the lowest glucose concentration used $(1.6 \mathrm{mmol} / 1)$, there was increased incorporation in both caffeine groups. However, in the caffeine/sucrose and sucrose pancreases, the glucose responsiveness of the process was markedly enhanced. The dose-response curve demonstrated an increased $V_{\max }$ for both caffeine groups and for sucrose. The sensitivity to glucose at the process of incorporation of ${ }^{3} \mathrm{H}$-leucine into proinsulin was significantly enhanced $(p<0.001)$ in the sucrose and caffeine/sucrose groups but not in the caffeine group, as reflected by glucose concentrations of $3.6 \pm$ $0.4,3.7 \pm 0.3,4.3 \pm 0.4$ and $4.8 \pm 0.4 \mathrm{mmol} / 1$ required to produce $50 \%$ of maximal stimulation in the sucrose, caffeine/sucrose, caffeine and control groups respectively.

\section{Discussion}

This investigation is the first report of neonatal pancreatic function in vitro after caffeine exposure in vivo and shows that maternal caffeine consumption has effects on the glucose responsiveness of the neonatal pancreas. The effects of caffeine are significantly modified by concurrent maternal sucrose supplementation.

Maternal caffeine consumption with or without sucrose supplementation produced a 'left' shift in the curve for glucose-induced insulin release from the neonatal pancreas. Sucrose supplementation of the caffeine-consuming dams produced an additional increase in the maximal insulin secretory response to glucose in the pancreases of the offspring. However, in physiological terms, the enhanced sensitivity of the release process attributable to caffeine exposure is likely to be more relevant, particularly as the calculated values for half-maximal stimulation indicate that the release process is extremely sensitive within the observed plasma glucose range of the neonate.

By contrast, the biosynthetic responsiveness of the neonatal pancreas to glucose was not enhanced by caffeine ingestion by the mother. Exposure to elevated plasma glucose levels in the offspring of dams receiving sucrose supplementation was accompanied by increased sensitivity of the biosynthetic process to glucose, irrespective of caffeine exposure. The observation that exposure to raised plasma glucose increases proinsulin synthesis is in agreement with recently reported observations of the effect of glucose administration in vivo on pancreatic function in neonatal rats [19]. The physiological relevance of the contrast in the effect of maternal caffeine ingestion on the process of insulin secretion compared with proinsulin biosynthesis by the neonatal pancreas is best appreciated when the neonatal glucose concentrations in vivo are considered. The calculated value for half-maximal stimulation of biosynthesis lies within the observed range of plasma glucose values for caffeine/ sucrose, sucrose and control animals but exceeds that of the offspring of dams ingesting caffeine without sucrose. This would explain the progressive depletion of pancreatic insulin stores and eventual hypoinsulinaemia observed in the caffeine group.

In the developing animal, conditions which deplete available insulin are associated with reduced body weight [20]. It is likely that early post-natal hypoglycaemia followed by depletion of insulin reserves in the absence of supplementary carbohydrate feeding contributes to the previously observed body size reduction in the caffeine-exposed infant rat [13].

The present investigation, showing a similar low threshold for insulin biosynthesis and release in the control group of neonatal offspring, is at variance with previous studies in both adult [9] and neonatal [3] rat islets in vitro. In those studies it was found that the glucose threshold of insulin biosynthesis was lower than that of insulin secretion, and that the half maximal response for glucose-stimulated insulin release was found in the glucose concentration range $7-8 \mathrm{mmol} / 1$ in islets from neonatal animals, compared with $4.1 \mathrm{mmol} / 1$ glucose in the control group in the present study. The enhanced sensitivity of the secretory process in our study is probably explained by the high concentration of amino acids in the 
incubation medium. These levels were chosen to replicate the physiological elevation of amino acids seen in the near-term [17] and neonatal rat [21]. Kervran and Randon [22] demonstrated that insulin release in response to glucose in the perifused fetal rat pancreas is potentiated by such elevated amino acid concentrations.

It is possible for the human infant to experience considerable methylxanthine exposure [12]. Caffeine consumption is usually unrestricted during pregnancy and early feeding even in pregnancies complicated by glucose intolerance. The extensive therapeutic use of the methylxanthines caffeine and theophylline in the management of neonatal apnoea constitutes another source of infant exposure [23].

The present investigation was not aimed at determining the mechanism of the caffeine effect in the pancreas. This may be due solely to cyclic AMP elevation following inhibition of cyclic AMP phosphodiesterase [24, 25] or to cyclic AMP independent effects on calcium mobilisation $[26,27]$. It is also possible that some of the observed effects resulted from altered supply of maternal fuels to the fetus or neonate, through effects on tissue lipolysis [28], rather than from direct exposure to caffeine. Irrespective of the mechanism involved, the observations may have implications relating to pancreatic function and glucose homeostasis in infants of mothers ingesting methylxanthines.

Acknowledgements. We thank the Royal Children's Hospital Research Foundation and the National Health and Medical Research Council for financial support of this work and Mr G. Gauchi for excellent care of the animals used in the study.

\section{References}

1. Lambert AE, Kanazawa Y, Burr IM, Orci L, Renold AE (1971) On the role of cyclic AMP in insulin release: I Overall effects in cultured fetal rat pancreas. Ann NY Acad Sci 185: 232-244

2. Heinze E, Skeinke J (1972) Insulin secretion during development: Response of isolated pancreatic islets of fetal, newborn and adult rats to theophylline and arginine. Hormone Metab Res 4: 234-236

3. Jarousse C, Rosselin $G$ (1975) Interaction of amino acids and cyclic AMP on the release of insulin and glucagon by newborn rat pancreas. Endocrinology 96: 168-177

4. Hellman B, Idahl LA, Lernmark A, Taljedal IB (1974) Pancreatic B cell recognition of insulin secretagogues: Does cyclic AMP mediate the effect of glucose. Proc Nat Acad Sci USA 71:3405-3409

5. Grill W, Cerasi E (1974) Stimulation by D-glucose of cyclic adenosine $3^{\prime}: 5^{\prime}$ monophosphate accumulation and insulin release in isolated pancreatic islets of the rat. J Biol Chem 249: 4196-4201

6. Malaisse WJ, Sener A, Koser M, Ravazzola M, Mallaisse-Lagae F (1977) The stimulus-secretion coupling of glucose induced insulin release: Insulin release due to glycogenolysis in glucose deprived islets. Biochem J 164: 447-454

7. Schatz H, Maier V, Hinz M, Nierle C, Pfeiffer EF (1973) Stimulation of $\mathrm{H}$-3-leucine incorporation into the proinsulin and insulin fraction of isolated pancreatic mouse islets in the presence of glucose, theophylline and cyclic AMP. Diabetes 22: 433-441

8. Malaisse WI, Pipeleers DG, Levy J (1974) The stimulus secretion coupling of glucose-induced release. XVI. A glucose-like and calcium-independent effect of cyclic AMP. Biochem Biophys Acta 362:121-128

9. Maldonato A, Renold AE, Sharp CWG, Cerasi E (1977) Glucose induced proinsulin biosynthesis: Role of islet cyclic AMP. Diabetes $26: 538-545$

10. Duran Garcia S, Jarrouse C, Rosselin G (1976) Biosynthesis of proinsulin and insulin in the newborn rat pancreas. Interaction of glucose, cyclic AMP, somatostatin and sulphonylureas on the ${ }^{3} \mathrm{H}$ leucine incorporation into immunoreactive insulin. J Clin Invest $57: 230-243$

11. Soyka LF, Neese AL (1978) Perinatal exposure to methylxanthines. Possible effects on pregnancy outcome. Clin Pharmacol Ther 23: 130

12. Brazier J-L, Renaud H, Ribon B, Salle BL (1979) Plasma xanthine levels in low birthweight infants treated or not treated with theophylline. Arch Dis Childh 54: 194-199

13. Dunlop M, Court JM (1981) Effects of maternal caffeine ingestion on neonatal growth in rats. Biol Neonat 39:178-184

14. Dunlop M, Court JM, Larkins RG (1981) The effects of maternal carbohydrate (sucrose) supplementation on the growth of offspring of pregnancies with habitual caffeine consumption. Biol Neonat 40: 196-198

15. Herbert V, Lau KS, Gottleib CW, Bleicher SJ (1965) Coated charcoal immunoassay of insulin. J Clin Endocrinol Metab 25: 1375-1384

16. McCoy TA, Maxwell M, Kruse PF (1959) Amino acid requirement of novitkoft hepatoma in vitro. Proc Soc Exp Biol Med 100: 115-118

17. Palou A, Arola L, Alemany M(1977) Plasma amino acid concentrations in pregnant rats and in 21 day foetuses. Biochem J 166: 49-55

18. Kemmler W, Steiner DF (1970) Conversion of proinsulin to insulin in a subcellular fraction of rat islets. Biochem Biophys Res Commun 41: 1233-1230

19. Gespach C, Jarousse C, Rosselin G (1979) Regulation of insulin biosynthesis in vivo by glucose in the newborn rat. Metabolism 28: 1046-1050

20. Portha B, Levacher C, Picon L, Rosselin G (1974) Diabetogenic effect of streptozotocin in the rat during the perinatal period. Diabetes 23: 889-895

21. Girard JR, Cuendet GS, Marliss EB, Kervran A, Rieufort AI, Assan R (1973) Fuels, hormones and liver metabolism at term and during early post-natal period in the rat. J Clin Invest 52:3190-3200

22. Kervran A, Randon J (1980) Development of insulin release by fetal rat pancreas in vitro. Effects of glucose, amino acids and theophylline. Diabetes 29:673-678

23. Aranda JV, Gorman W, Loughnan PM, Aldridge A (1978) Pharmacokinetic profile of caffeine in the premature infant with apnoea. J Paediatr 94: 663-668

24. Sams DJ, Montague W (1972) The role of adenosine $3^{\prime}: 5^{\prime}$ cyclic monophosphate in the regulation of insulin release. Properties of islet-cell $3^{\prime}: 5^{\prime}$ cyclic monophosphate phosphodiesterase. Biochem J 129: 945-952

25. Ashcroft SJ, Randle PJ, Taljedal IB (1972) Cyclic nucleotide phosphodiesterase activity in normal mouse pancreatic islets. FEBS Lett 20: 263-266

26. Formby B, Capito K, Egeberg J, Hedeskov CJ (1976) Ca activated ATP-ase activity in subcellular fractions of mouse pancreatic islets. Am J Physiol 230: 441-444

27. Dunlop M, Larkins RG, Court JM (1981) Methylxanthine effects on cyclic adenosine $3^{\prime}: 5^{\prime}$ monophosphate phosphodiesterase activity in preparations of neonatal rat cerebellum: Modifications by trifluoperazine. Biochem Biophys Res Commun 98: 850-857

28. Bellet S, Kershbaum A, Finck EM (1968) Response of free fatty acids to coffee and to caffeine. Metabolism 17:702-707

Received: 9 November 1981

and in revised form: 24 May 1982

\section{Dr. M.E. Dunlop}

Department of Medicine

University of Melbourne

Repatriation General Hospital

Heidelberg, 3081, Victoria

Australia 